\title{
Clinical utility of three sensory modulation measures for the child with ASD in South Africa: A practitioner's perspective
}

\author{
Kerry Lee Evetts, B. OT (UKZN); M. OT (UKZN). http://orcid.org/0000-00 I-55 I 7-97 I 2 \\ Practice Owner and Senior Clinician, Kerry Evetts Occupational Therapy, Varsity Lakes, Queensland, Australia.
}

\section{*Gina Rencken, B. OT (UP); M. OT (UFS). http://orcid.org/0000-0002-3658-4453}

Lecturer, Discipline of Occupational Therapy, College of Health Sciences, University of KwaZulu Natal (Westville Campus), Durban, South Africa.

\author{
Pragashnie Govender, B. OT. (UKZN); M. OT (UKZN); PhD (UKZN). \\ http://orcid.org/0000-0003-33 I5-3743 \\ Associate Professor, Discipline of Occupational Therapy, College of Health Sciences, University of KwaZulu Natal (Westville Campus), \\ Durban, South Africa.
}

Background: There is need for the assessment of sensory modulation in children with Autism Spectrum Disorder (ASD) to be culturally, environmentally, economically and language sensitive to the South African population. It is assumed that the measures in current use are not appropriate, acceptable, practical or accessible for the South African child with ASD.

Aim: This study sought the practitioners' perspective on the clinical utility of three sensory modulation measures for children diagnosed with ASD in South Africa.

Methods: A quantitative survey method was used to collect data from a purposive sample of 3 I SAISI Occupational Therapy members nationwide.

Results: Urban participants accounted for $83 \%$ of the sample, $80 \%$ were in private practice and $67 \%$ from English-speaking practices. All three measures demonstrated some level of clinical utility. The Sensory Processing Measure (SPM) was found to be more appropriate than the Sensory Profile (SP) $(p<0.007)$, although the SP was more accessible than the SPM $(p<0.002)$. Amongst non- English speakers the Sensory Experiences Questionnaire (SEQ) was more acceptable than the SPM $(p<0.045)$.

Recommendations: The SPM should be used in an interview format, with recommended changes and translation into the caregivers' home language, until a South African specific sensory screening measure, which can be readily reproducible in several local languages, is developed.

Keywords: Sensory Profile, the Sensory Processing Measure, Sensory Experiences Questionnaire, culturally sensitive, language appropriate

\section{INTRODUCTION}

There is an identified gap in the provision of South African validated assessment tools for children diagnosed with autism spectrum disorder (ASD) $)^{1,2}$. One of the behavioural symptoms associated with ASD and listed in the DSM-5, is 'unusual interest in sensory features of the environment' or 'reactivity (hypo- or hyper) to sensory stimulation's. Sensory reactivity or modulation is defined as the level of a behavioural response to a sensation. It includes the ability to adjust and organise one's own behavioural and emotional reactions to stimuli. There is extensive research estimating the number of children with ASD who experience unusual responses to sensory experiences ${ }^{4-6}$, with as high as $95 \%$ of these children experiencing sensory processing difficulties ${ }^{4}$. Given that individuals of all ages diagnosed with ASD present with varied sensory symptoms compared to age peers ${ }^{7}$, it becomes necessary to consider appropriate instruments for the assessment of sensory modulation.

Caregiver-report measures of sensory modulation, meet the requirements for being, relatively inexpensive to administer, com- pleted within a short timeframe and used in assessing children diagnosed with ASD. There are a number of instruments that measure sensory modulation in young children based on caregiver-reports. These include the Sensory Profile (SP) ${ }^{8}$, the Sensory Processing Measure (SPM $)^{9}$ and the Sensory Experiences Questionnaire $(\mathrm{SEQ})^{10}$. However, all three instruments have been designed and normed in the USA. There are currently no ASD-specific measures to assess sensory modulation in the multi-cultural South African context. Any such instrument would need to recognise language and cultural differences, but still yield clinically sensitive information about the individual child with ASD, which will be used to structure an intervention programme. As developing such tools would be costly and time consuming ", three caregiver-report, sensory modulation measures were evaluated for use with the South African child with ASD.

\section{LITERATURE REVIEW}

Jean Ayres identified patterns of sensory integrative disorders, 
including difficulties with sensory reactivity/modulation, sensory discrimination and perception, vestibular processing and dyspraxia ${ }^{12}$. An atypical response to sensory experiences or situations is referred to as "sensory modulation dysfunction" (SMD), and may be characterised by over-responsiveness, under-responsiveness, or excessive seeking or avoiding ${ }^{12}$. Since sensory differences are often predictive of maladaptive behaviours in $\mathrm{ASD}^{13}$, accurate assessment and appropriate intervention of sensory reactivity/modulation may have a profound impact on the functional performance of children with ASD in South Africa.

A systematic review of assessment tools currently available for identifying different patterns of sensory processing in children aged three to II years was carried out in $2017^{14}$. This review revealed that the most commonly used tools included the Sensory Integration and Praxis Test $\left(\right.$ SIPT) ${ }^{15}, \mathrm{SP}^{8}$ and SPM ${ }^{9}$. There are currently six versions of SPM (English, Danish, Finish, Swedish, Norwegian, and Chinese) and six versions of SP (English, Spanish, Arabic, Turkish, Indian, and Chinese) ${ }^{16}$. Occupational therapists plan interventions based on evaluation by interpreting data from these assessment tools that includes gathering information from the caregiver and using observation of the child ${ }^{16}$. Baranek and colleagues ${ }^{6}$ report that caregiver-report instruments are ecologically valid for obtaining information about children's behaviour. In the South African context, they are considered a good choice from a cost and time feasibility perspective.

However, with a population of 59 million in South Africa, II official languages and a wide range of cultures ${ }^{17}$, sourcing measures developed in the USA raise issues when used with children with ASD in South Africa. Factors that need to be considered in instrument choice are the children and caregiver's culture and language, as well as bias and equivalence of the instrument. The culture within which a child is raised influences values, interactions, social customs, and family structures that help organise their environment $^{15}$. Cultural fairness stipulates that items on tests must not prejudice the performance of children of same ability from different backgrounds due to the unfamiliarity of tasks, and therapists need to be mindful of the influence of culture on sensory processing when planning intervention ${ }^{18,19}$. In 2015 , eight out of ten households in South Africa were headed by black Africans $(80,41 \%)^{17}$ who are likely to be second or third language English speakers which may impact on the use of these assessments. Thus, attention to language differences is important and at times result in the instrument not being used as a caregiver-report tool. Answers also need to be interpreted with care ${ }^{20-22}$.

Interpersonal sensitivity and a good cross-cultural understanding and communication skills are needed in order to minimise potential bias in the administration of any test ${ }^{23}$. A significant concern is the use of norms for interpretation, where the cultural populations are not homogeneous. This raises the question of the validity of imported assessments in the South African context ${ }^{24}$. Previous research shows that different cultural/ethnic groups perform differently with the test version designed in their own home language ${ }^{25}$.

\section{Assessing Clinical Utility}

Smart ${ }^{26}$ suggested a multi-dimensional model to assess the clinical utility of a test. His model uses dimensions of appropriateness, accessibility, practicability and acceptability (Table I)

Clinical utility, for the purposes of this study, was defined by the authors as the extent to which the identified sensory reactivity/modulation instruments assist occupational therapists in South Africa, to accurately diagnose the sensory profile of a child with
Table I: Summary of the dimensions of clinical utility (adapted from Smart ${ }^{26}$ )

\begin{tabular}{|l|l|l|}
\hline DIMENSIONS & ASPECTS & $\begin{array}{l}\text { ISSUES TO BE } \\
\text { CONSIDERED }\end{array}$ \\
\hline APPROPRIATE & $\begin{array}{l}\text { Effective } \\
\text { Relevant }\end{array}$ & $\begin{array}{l}\text { Existence of formal evi- } \\
\text { dence. } \\
\text { Impact on existing } \\
\text { treatment processes. } \\
\text { Importance for clinical } \\
\text { decision making. }\end{array}$ \\
\hline ACCESSIBLE & $\begin{array}{l}\text { Resource } \\
\text { Implications } \\
\text { Procurement }\end{array}$ & $\begin{array}{l}\text { Costs and cost } \\
\text { effectiveness. } \\
\text { Availability, supply and } \\
\text { quality. } \\
\text { Financial processes. }\end{array}$ \\
\hline PRACTICABLE & $\begin{array}{l}\text { Functional } \\
\text { Suitable } \\
\text { Training/ } \\
\text { Knowledge }\end{array}$ & $\begin{array}{l}\text { Are the materials, supplies } \\
\text { etc available and working? } \\
\text { Everyday constraints on } \\
\text { training. }\end{array}$ \\
\hline ACCEPTABLE & $\begin{array}{l}\text { To clinicians } \\
\text { To clients } \\
\text { To society }\end{array}$ & $\begin{array}{l}\text { Ethical, legal, social or } \\
\text { psychological concerns } \\
\text { that may affect practice, } \\
\text { treatment processes and } \\
\text { acceptance. }\end{array}$ \\
\hline
\end{tabular}

ASD, and thereby prescribe appropriate therapy plans for them, within the cultural, social and developmental needs of the child.

There is an identified gap in the provision of culturally sensitive, language appropriate sensory reactivity/modulation measures for ASD children in South Africal, 2 . This study, undertaken for degree purposes, focused on exploring the practitioners' perspective on whether three caregiver-report, sensory reactivity/modulation measures have any clinical utility for a child with ASD in South Africa.

\section{METHODS}

An electronic survey, using a cross-sectional descriptive design was used to (i) establish the occupational therapy practitioner's perspective on the clinical utility (appropriateness, accessibility, practicability and acceptability) of three sensory reactivity/modulation measures (SP, SPM, SEQ) for the South African child with ASD, (ii) ascertain the challenges of using caregiver-report sensory reactivity/modulation measures; and (iii) to establish particular items of the measures that were problematic.

\section{Population and sampling}

Non-probability purposive sampling was used to recruit participants from the national database of 850 occupational therapists registered with the South African Institute of Sensory integration (SAISI). Participants had to have completed the South African Sensory Integration Course on test interpretation (SASIC3), had to have assessed children with ASD in their career and had to be working within an ASI frame of reference.

\section{Research instrument}

The literature review on ASI, ASD and clinical utility provided the background for the creation of the survey. The first section of the survey required participants to complete demographic information. An open-ended question sought information on challenges participants had experienced when using sensory modulation measures in their current setting. This was followed by 16 four-point Likert scale questions, in the four clinical utility domains of appropriate- 
ness, accessibility, practicability and acceptability ${ }^{25}$. The Likert scale ranged from I= strongly disagree, $2=$ disagree, $3=$ agree, to 4 $=$ strongly agree and was designed to force a positive or negative response from the participant. To be appropriate according to the clinical utility definitions, an instrument should yield results that can be used in clinical decision-making and impact on the child's treatment plan. To be considered accessible, it must be readily available in the work place, and the caregiver should be able to use it as a self-report measure. For the instrument to be practicable it should be functional and suitable for its use in the participants setting. For the instrument to be acceptable, it should be ethically sound, socially and culturally appropriate to both the target population and the practitioner. The final section of the survey yielded responses to open ended questions on the specific items from each sensory modulation measure that were considered problematic when used in their setting. The data collection phase spanned eight weeks, with reminders sent after three, five and seven weeks.

The following three Sensory Reactivity/Modulation Tools were included for evaluation in the survey:

\section{The Sensory Profile (SP)}

The SP assesses the responses of children over 125 commonly occurring daily experiences, with caregivers rating the frequency with which their child responds to these on a five-point Likert scale "always", "frequently, “occasionally", "sometimes”, "never”. The I 25 items are classified into eight categories: Auditory, Visual, Activity Level, Taste or Smell, Body Position, Movement, Touch, and Social/Emotional ${ }^{8}$.

\section{The Sensory Processing Measure (SPM)}

The SPM home form is caregiver rated on a four-point Likert scale, with 75 questions relating to a variety of situations relating to the child's sensory processing ability, praxis ability and social participation that may impact a child's performance in school, home, and community settings. The descriptors for the Likert scale in this instrument are "never", "occasionally", "frequently" and "always".

\section{The Sensory Experiences Questionnaire (SEQ)}

The SEQ is a 05 item caregiver-report tool, measuring the child's behavioural responses to sensory stimuli naturally occurring in daily life, sensory behaviours across sensory response patterns of hypo-responsiveness, hyper-responsiveness, sensory seeking, and enhanced perception as well as auditory, visual, tactile, gustatory, and vestibular modalities in social and non-social contexts ${ }^{10}$. The frequency of the child's response on first 97 items is based on a five-point Likert scale ranging from "almost never" to "almost always". The last eight items allow the caregiver to elaborate on the child's sensory behaviour ${ }^{10}$.

\section{Pilot Study}

Reliability of the survey questionnaire was established through the pilot study where eight expert ${ }^{27}$ occupational therapists reviewed and commented on the questions and the rating scale used. Content validity was achieved by carefully describing the ASD child in the South African context and including items that represented the issues raised in the literature review and subjecting those domains to expert scrutiny ${ }^{27}$ via the pilot study. Construct validity was observed by combining ASI and clinical utility theory to inform the design of the survey questions. The survey questionnaire was thereby designed to access both theoretical constructs through its scaled scores in order to derive clinically relevant information from the subsequent data. Feedback resulted in editing and rewording of items, changes to the layout and presentation of questions and the inclusion of the open-ended question on items that were found to be problematic in practice.

\section{Data Collection}

An invitation letter was distributed to the members of SAISI via the administrator. Those who agreed to participate, provided they met the inclusion criteria, were forwarded the survey by the principal author. Sampling bias may have been present as the therapists who met the criteria and responded to the invitation to participate, automatically became part of the final sample $(n=3 I)$. Since the invitation email was sent to all members on the SAISI database, every registered SAISI member had an equal opportunity to respond provided they met the specific inclusion criteria.

\section{Data Analysis}

Demographic information was entered onto a Microsoft Excel 2007 spreadsheet and descriptive statistics were used to analyse the frequencies of responses. The open-ended questions on challenges experienced was analysed thematically ${ }^{28}$ to establish the main themes and the frequency of these was presented using descriptive statistics. The 16 questions in four dimensions of clinical utility ${ }^{26}$ were transferred onto a spreadsheet and descriptive statistics used to establish which sensory reactivity/modulation measure held the most clinical utility, according to each of the four dimensions, as well as an overall measure. Descriptive statistics were used to calculate the mean score for clinical utility for each measure and statistically significant differences between the clinical utility was established using t-tests. T-tests were also used to establish whether differences existed between overall clinical utility and the years of experience, setting and language of the clients. This was done to determine which, if any, measure had higher clinical utility within these linguistic/cultural/ environmental factors ${ }^{27}$. The level of significance was set at $\mathrm{p} \leq 0.5$.

\section{*Ethics}

Full ethical approval was granted from University of KwaZulu-Natal (Ethical Clearance number: HSS/0409/0 I 7M). The HPCSA guidelines for ethical good practice were observed during all aspects of this study ${ }^{29}$. A process of informed consent was followed during piloting and data collection. All data participants were de-identified and aggregated during analysis, thereby maintaining anonymity and privacy of participants.

\section{RESULTS}

Practitioners who responded were experienced with $65 \%(n=20)$ having more than 10 years' experience as an occupational therapist. The majority (67\%; $n=21$ ) of practitioners consulted were English- speaking clients, worked mainly in private practice settings $(80 \% ; n=25)$ and most $(83 \% ; n=26)$ were based in urban areas.

\section{Clinical Utility of the three Measures Overall Mean Scores}

Overall mean scores for clinical utility of the SP, SPM and the $S E Q$ demonstrated no statistically significant difference $(p \geq 0.5)$. The mean rating was a ' 3 ' on the Likert scale which indicated 


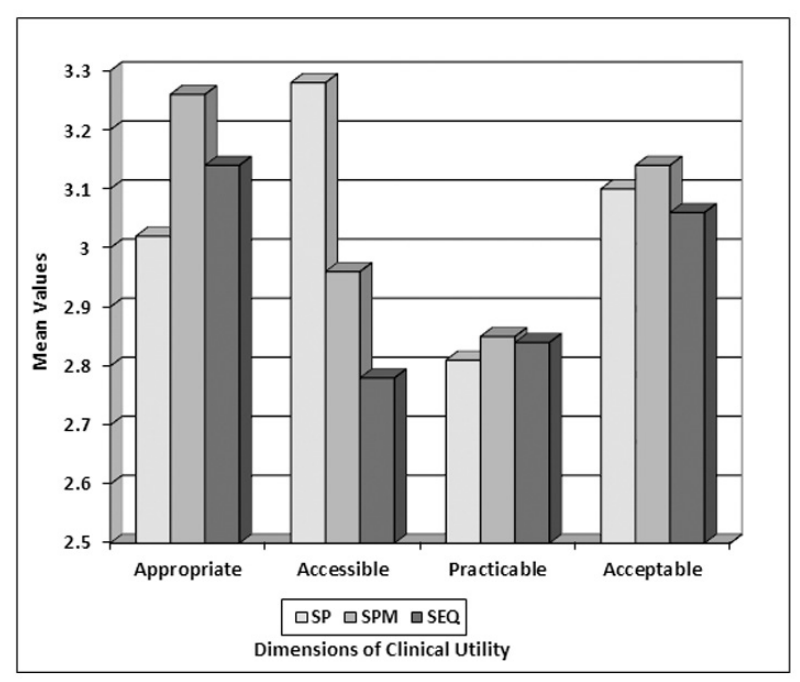

Figure I: Overall mean scores for clinical utility of the three measures.

agreement with the three measures' clinical utility. The three measures were then analysed for statistical significance $(p<0.5)$ over each of the dimensions of clinical utility, which included appropriateness, accessibility, practicability and acceptability (Figure I).

\section{Clinical Utility: Appropriateness}

A clinically significant difference between the SP and SPM was noted, in favour of the SPM $(p=0.007)$. This was consistent when compared to practitioner experience and geographical location. However, with the group of non-English speaking or second language English speakers, the SEQ held significantly higher utility than the SP $(p=0.01)$ and SPM $(p=0.02)$.

\section{Clinical Utility: Accessibility}

Statistically significant differences between the three measures were noted with the SP being most accessible. The SP was more accessible than the SPM $(p=0.002)$, and the SEQ $(p=0.00 I)$ and the SPM was more accessible than the SEQ $(p=0.03)$. The majority $(90 \% ; n=28)$ of the practitioners had access to the SP within their work place.

\section{Clinical Utility: Practicability}

There were no significant differences between any of the measures for practicability. Cost of the measures was a concern cited by some practitioners, with $32 \%(n=10)$ reporting the cost was too high. Other practicability measures indicate $13 \%$ found the questionnaires too long, $10 \%$ found the rating scales challenging to use and $10 \%$ reported the layout was not easy to follow.

\section{Clinical Utility: Acceptability}

There were no significant differences between the three measures for acceptability. A small number of practitioners (22\%; $\mathrm{n}=7$ ) commented that the measures were not relevant for the child with ASD in South Africa. A statistically significant preference for the SEQ $(p=0.020)$ over the SPM was seen amongst the non-English speaking or second language English speakers with a significant preference of the SPM over the SEQ $(p=0.009)$ noted in English speakers.
Challenges in use of caregiver-report Sensory Reactivity/ Modulation Measures

Caregiver-report sensory reactivity/modulation measures were used by $90 \%(n=28)$ of the participants, with $68 \%(n=19)$ of them using the SP, I8\% $(n=5)$ using the SP and SPM, and I4\% $(n=4)$ using only the SP. Of the 28 participants that used the measures, $27(97 \%)$ reported challenges in the use with children with ASD.

The themes that emerged included parents' lack of insight into their child's behaviours ( $40 \%, n=I I)$, parents having difficulty understanding the measure due to ambiguity $(74 \%, n=20)$, language barriers to using the measure $(22 \%, n=6)$, the measure being too long $(30 \%, n=8)$ or misused (over-or understate the child's difficulties) $(26 \%, n=7)$, denial of the child's diagnosis $(18 \%, n=5)$ and that interviews with the parents and/or observation of the child $(37 \%, n=10)$ are often needed to supplement the information given in the sensory reactivity/modulation questionnaire.

\section{Problematic Items on the three Measures}

This was an optional section of the survey with and 22 participants providing responses. These included suggestions for changes in wording, phrases, task items, cultural and environmental specific changes as well as ASD core deficit comments. Particular reference was made for the use of SPM in South Africa as a caregiver-report measure for literate, first language English speakers.

Participants noted that in South Africa, the SPM can guide an interview with a caregiver to gain useful information about the child's responses to sensory input in their environment. For valid and reliable information, the caregiver's home language is preferred and analysis of items should consider cultural norms. When used with these considerations, final scores can only be used as a guide as the deviations from the normed scores threaten the validity of the measure. The considerations for the South African context from the practitioners in this study appear in Table II (p26).

\section{DISCUSSION AND IMPLICATIONS OF THE STUDY}

Considering the lack of clinical utility studies of sensory reactivity/ modulation measures, the results of this study are significant. This is in relation to practitioners' perspectives on clinical utility that will inevitably influence how sensory reactivity/modulation measures are used when assessing children with ASD in South African private practice settings. The participants were largely from English speaking, private practices located in urban areas which may have skewed the results. No single test held overall higher clinical utility than the others, and the overall rating indicated agreeability with the clinical utility of the measures as assessed across the four dimensions in this study. However, on analysis of each dimension and comparison to the data from different demographic groups, some significant findings emerged.

In establishing clinical utility appropriateness, the SPM was indicated to be more appropriate for an ASD population due to the social participation section, communication items and focus on activities of daily living (ADLs). The SEQ was considered more appropriate for the non-English speaking or second language English group, as it was short, concise, and focused on ASD-related questions. Regarding the clinical utility accessibility dimension, the SP was the most utilised measure and accessible in most of the workplaces of participants. The SEQ is not yet widely known or available in SA and therefore held the least accessibility. However, it was noted that it was easy to understand and would be used if available.

Clinical utility practicability was related to the cost of the measure and the length of time taken to complete the measures, 
Table II: Considerations in the administration of the SPM within the South African Context

\begin{tabular}{|c|c|c|c|c|}
\hline LANGUAGE & TASKS & CULTURE & ENVIRONMENT & CORE ASD DEFICIT \\
\hline $\begin{array}{l}\text { If a word or phrase } \\
\text { is not understood, } \\
\text { clarify the meaning by } \\
\text { providing a more com- } \\
\text { monly used word in that } \\
\text { language or provide an } \\
\text { explanation. } \\
\text { The following words are } \\
\text { anticipated to require } \\
\text { explanation: } \\
\text { unappealing exces- } \\
\text { sively fearful imitate } \\
\text { distressed appropriate } \\
\text { seek strobe light fluores- } \\
\text { cent light brassy shrill pet } \\
\text { animals } \\
\text { For item } 33 \text { use an ex- } \\
\text { ample of hugging others } \\
\text { if confused by the word } \\
\text { 'touch'. }\end{array}$ & $\begin{array}{l}\text { Item 29: Exposure to } \\
\text { "flutes and trumpets" } \\
\text { may be limited so per- } \\
\text { haps the example of a } \\
\text { whistle or vuvuzela. } \\
\text { Item 46,49: Use of } \\
\text { "pencil" or "spoon" or } \\
\text { "legos" (item 73) may } \\
\text { vary according to cul- } \\
\text { ture and exposure. } \\
\text { Item } 56 \text { : "Teeter-tot- } \\
\text { ter" is a see-saw. } \\
\text { Item } 58 \text { : "Curb" should } \\
\text { be worded as pave- } \\
\text { ment. }\end{array}$ & $\begin{array}{l}\text { Throughout: "Interact/ partici- } \\
\text { pate appropriately" could mean } \\
\text { different things in different } \\
\text { cultures so caution should be } \\
\text { used. } \\
\text { Item 4: The concept of personal } \\
\text { space differs culturally. } \\
\text { Item 5: "Eye contact" can be } \\
\text { open to interpretation accord- } \\
\text { ing to one's cultural norms. } \\
\text { Item 7: may not be appropriate } \\
\text { in some cultures who prefer } \\
\text { quiet mealtimes. } \\
\text { Item } 42 \text { : uses cooked spinach as } \\
\text { an example which may be of- } \\
\text { fensive to some. }\end{array}$ & $\begin{array}{l}\text { May not have exposure } \\
\text { to light switches (Item 19), } \\
\text { hairdryers/vacuum cleaners } \\
\text { (item 22), air conditioner/ } \\
\text { lawn mower (item 27) } \\
\text { in the home. Use other } \\
\text { examples. } \\
\text { Item 56. May not have } \\
\text { playground equipment } \\
\text { available. } \\
\text { Item 65: Many may not } \\
\text { be exposed to "escalators/ } \\
\text { elevators" and "flushing } \\
\text { toilets". }\end{array}$ & $\begin{array}{l}\text { Twelve of the } 74 \text { items } \\
\text { were noted by partici- } \\
\text { pants to be as a result of } \\
\text { core ASD deficits and not } \\
\text { necessarily of sensory } \\
\text { origin. } \\
\text { These are items number: } \\
1,2,3,5,8,9,10,72 \\
\& 73 \text {. Please use cau- } \\
\text { tion when interpreting } \\
\text { answers. }\end{array}$ \\
\hline
\end{tabular}

the layout and the rating scale. The practicability results remained consistent between all three measures, across all four dimensions. The practicability scores were generally the lowest scores, from all four dimensions of clinical utility assessed, indicating overall dissatisfaction with all of the measures for their practical use. Clinical utility acceptability explored whether the practitioner would use the measure in their workplace. They answered questions about the relevance of the measures culturally, environmentally and diagnostically. Results indicate and possibly guide decision making in choice of measures, with the SPM being more relevant to the English-speaking client population, and the SEQ being more relevant to the non-English speaking population.

The findings of this study were similar to those seen in other cross-cultural studies with sensory reactivity/modulation measures. Israeli ${ }^{10}$, Spanish ${ }^{18}$ and Arabic ${ }^{19}$ studies found that language barriers and cultural and environmental differences impacted the validity of the measures in cultures that differed from the USA. An Australian study ${ }^{30}$ found the SP to be valid in their context, which is likely due to the official language being English. Their cultural differences in the study were considered minor, and was catered for by using suggested alternative phrases/words in a few places when administering the measure in Australia. South Africa is a multi-lingual country, with vast diversity in culture, socio-economic status and environments. Although this influences the validity of sensory reactivity/modulation measures, full-scale translation and psychometric testing of sensory reactivity/modulation measures are not feasible in this context. Many participants commented that an electronic measure which can be emailed to parents, is easy to understand, translatable, not too long, scored automatically, and is reasonably priced would be favoured. The SP and SPM are both available online for a fee.

Benefits of the measures are highlighted in this study even though literacy levels and language differences in South Africa present challenges with using these caregiver-report measures. It is clear that caregiver-report sensory reactivity/modulation measures are considered valuable by the number of therapists already using them. The therapists do adapt to these challenges, by using a sensory reactivity/modulation measure as a guide to parent interviews, and used both a parent interview and observation of the child as part of their assessment in ASD, before planning an intervention. Despite the SP being the most utilised of the sensory reactivity/modulation measures in this sample (holding the highest clinical utility for 'accessibility') the SPM and SEQ demonstrated higher clinical utility than the SP in the 'appropriateness' and 'acceptability' dimensions, for the child with ASD in South Africa. Comments included finding the SPM more suitable for ASD due to the social participation, and ADL questions. The SEQ was favoured also due to the 'fascinations' sections of the questionnaire which many felt was appropriate for ASD. It was described to be shorter and easier to complete when compared to the SP and SPM. The SP had many more negative comments including being lengthy, daunting to parents, having complex questions, taking a long time to score and having too many gaps in assessing ADL for the child with ASD.

Limitations of the study include the majority of the study participants being practitioners in private practice in urban settings. Since the inclusion criteria required the practitioner to have training to SASIC level 3, it highlights that this training appears to be accessible to those in private practice. The training is however, inaccessible geographically to rural occupational therapists, as there is only one course per year and is usually presented in an urban setting.

\section{RECOMMENDATIONS}

This study highlights that the internationally, more specifically USAdeveloped sensory reactivity/modulation measures do not have adequate clinical utility, in all dimensions, for caregivers of children with ASD in South Africa, that may very well bias the validity of the measures. However, the majority of participants do use sensory reactivity/modulation measures as part of their ASD assessment. It is thus clear that these measures do hold some value, even though adaptations may be required in administration and interpretation. The release of the online scoring versions for the SPM and SP have made access to practitioners less financially burdensome.

In clinical practice, guidance on the choice of instrument for their client population (SPM for English language speakers, SEQ for non-English language speakers), and using the instruments to guide interview questions and assisting in parent/caregiver understanding of items are important. Further research is however recommended, 
with a particular focus on rural contexts with non-English first language caregivers. There may be a need for a sensory reactivity/ modulation measure, in additional languages, with terminology and phrases suitable for the culture in that language group. The measure should be concise and readily available at low cost and used thereafter to guide parent interviews and intervention. Those practitioners currently practicing sensory integration occupational therapy with children with ASD, and whose caregivers are not first language English speakers, should administer the SPM with some adaptations.

Tertiary educators and trainers in sensory integration should consider teaching the SPM with recommended adaptations until such time a South African tool becomes available. The process of culturally and linguistically fair occupational therapy assessments in South Africa should be reviewed, and research and development of such supported.

\section{CONCLUSIONS}

The literature is clear on the need for ASD assessment to be fair and just, culturally sensitive and environmentally suitable, for any such assessments to hold validity. The results of this study indicate that whilst the measures hold clinical utility for some of the South African population, this is the minority. Specifically, the sensory reactivity/modulation measures normed in the USA hold limited clinical utility for non- or second language English speaking caregivers of ASD children. In this study, practitioners reported that sensory reactivity/modulation measures are more beneficial in the caregivers' home language, and that instruments should be culturally and environmentally applicable. A sensory reactivity/modulation measure for children with ASD thus needs to be developed. The practice implication for this in occupational therapy indicates careful thought and clinical reasoning in choosing assessment instruments, specifically with the non-English speaking child or caregiver and in rural settings.

\section{AUTHOR CONTRIBUTIONS}

Kerry Lee Evetts conducted the research and was the primary author of the manuscript. Gina Rencken and Pragashnie Govender supervised the study and assisted in the conceptualisation, analysis and interpretation of the data and in revising the manuscript through the review process.

\section{Declaration of Conflict}

The authors declare no conflict of interest.

\section{REFERENCES}

I. Smith L, Malcolm-Smith S, de Vries PJ. Translation and cultural appropriateness of the Autism Diagnostic Observation Schedule-2 in Afrikaans. Autism [Internet]. SAGE Publications; 2016 May 25;2I (5):552-63. Available from: http://dx.doi.org/10.1 I 77//36236/316648469

2. Van Jaarsveld A, Mailloux Z, Herzberg DS. The use of the Sensory Integration and Praxis tests with South African children. South African Journal of Occupational Therapy. 2012;42(3): 12-18.

3. American Psychiatric Association. Diagnostic and statistical manual of mental disorders (DSM- $5^{\circledR}$ ). American Psychiatric Pub; 2013.

4. Dickie VA, Baranek GT, Schultz B, Watson LR, McComish CS. Parent Reports of Sensory Experiences of Preschool Children With and Without Autism: A Qualitative Study. American Journal of Occupational Therapy [Internet]. AOTA Press; 2009 Mar I;63(2): I 72-8I. Available from: http://dx.doi.org//0.5014/ajot.63.2.172
5. Tomchek SD, Dunn W. Sensory Processing in Children with and without Autism: A Comparative Study Using the Short Sensory Profile. American Journal of Occupational Therapy [Internet]. AOTA Press; 2007 Mar I;61 (2): 190-200. Available from:

http://dx.doi.org//0.5014/ajot.61.2.190

6. Baranek GT, David FJ, Poe MD, Stone WL, Watson LR. Sensory Experiences Questionnaire: discriminating sensory features in young children with autism, developmental delays, and typical development. Journal of Child Psychology and Psychiatry [Internet]. Wiley; 2005 Sep 22;47(6):59I-60I. Available from: http://dx.doi.org/ I0. I I I I/j. I469-7610.2005.01546.x

7. Ben-Sasson A, Hen L, Fluss R, Cermak SA, Engel-Yeger B, Gal E. A Meta-Analysis of Sensory Modulation Symptoms in Individuals with Autism Spectrum Disorders. Journal of Autism and Developmental Disorders [Internet]. Springer Science and Business Media LLC; 2008 May 30;39(I): I-II. Available from: http://dx.doi.org// 0.1007/s 10803-008-0593-3

8. Dunn W. Sensory profile. San Antonio, TX: Psychological Corporation; 1999.

9. Parham LD, Ecker C. Sensory processing measure (SPM). Western Psychological Services; 2007

10. Baranek GT. Sensory experiences questionnaire version 3.0. Unpublished manuscript.

II. Franz, L., Chambers, N., Von Isenburg, M., De Vries, P.J., 2017. Autism spectrum disorder in sub-saharan africa: A comprehensive scoping review. Autism Research 10(5): 723-749. https://dx.doi.org/10.1002\%2Faur. 1766

12. Schaff, R., Schoen,S., Smith Roley, S.,Lane, S., Koomar, J., MayBenson, T. A Frame of Reference for Sensory Integration IN Kramer, P \& Hinojosa, J Frames of References for Pediatric Occupational Therapy 3rd edition.20 I0: pI II-II2

13. Lane AE, Young RL, Baker AE, Angley MT. Sensory processing subtypes in autism: Association with adaptive behavior. Journal of Autism and Developmental Disorders. 2010;40(1): I I2-122. https://doi.org/10.1007/s 10803-009-0840-2

14. Jorquera-Cabrera S, Romero-Ayuso D, Rodriguez-Gil G, TriviñoJuárez J-M. Assessment of Sensory Processing Characteristics in Children between 3 and II Years Old: A Systematic Review. Frontiers in Pediatrics. 2017; 5-57. https://doi.org/10.3389/fped.2017.00057.

15. Ayres AJ. The Sensory Integration and Praxis Tests. Los Angeles: Western Psychological Services. 1989.

16. Windsor M, Smith-Roley SS, Szklut S. Assessment of Sensory Integration and Praxis. In: Roley SS, Blanche El, Schaaf RC, editors. Understanding the nature of sensory integration with diverse populations. Communication Skill Builders; 2001:215.

17. Statistics South Africa. P03 10 - Living Conditions of Households in South Africa, 20I4/20I5. Available from https://www.statssa.gov.za/publications/P03 10/P03 I 020 I 4.pdf Accessed I4 June 202 I

18. Caron KG, Schaaf RC, Benevides TW, Gal E. Brief Report-CrossCultural Comparison of Sensory Behaviors in Children with Autism. American Journal of Occupational Therapy. 2012; 66(5): e77-80. https://doi.org/10.5014/ajot.2012.004226

19. Peña ED. Lost in translation: Methodological considerations in crosscultural research. Child Development. 2007 Jul I;78(4): I 255- 1264. https://doi.org/10.1 I I I/j. I467-8624.2007.01064.x

20. Román-Oyola R, Reynolds SE. Validating the response process of the Spanish version of the Short Sensory Profile: A pilot study using cognitive interviews. Journal of Occupational Therapy, Schools, \& Early Intervention. 2010;3(3):197-206. 
https://doi.org/10.1080/194II243.2010.515189

21. Almomani FM, Brown C, Dahab SA, Almomani M, Nadar M. Cross cultural adaptation of the adolescent/adult sensory profile: establishing linguistic equivalency and psychometric properties of the Arabic version. Disability and Rehabilitation. 2014;36(9):765-770.

https://doi.org/10.3109/09638288.2013.819386

22. Brown C, Dunn W. Adolescent/Adult Sensory Profile manual. San Antonio, TX: Psychological Corporation. 2002.

23. HPCSA. Framework for Education, Training, Registration and Scope for Psychometry 2019. Available from

https://www.hpcsa.co.za/Uploads/PSB_2019/Exams/Form_94_Psychometry_Framework_for_Education\%20_Training_Registration_and_Scope_for_Psychometry.pdf Accessed I 4 June 2021 .

24. He J, van de Vijver F. Bias and Equivalence in Cross-Cultural Research. Online Readings in Psychology and Culture [Internet]. Grand Valley State University Libraries; 2012 Jun I;2(2). Available from: http://dx.doi.org/10.9707/2307-0919.1 I I I

25. Malda M, van de Vijver FJR, Temane QM. Rugby versus Soccer in South Africa: Content familiarity contributes to cross-cultural differences in cognitive test scores. Intelligence [Internet]. Elsevier BV; 2010 Nov;38(6):582-95. Available from: http://dx.doi.org/10.1016/j.intell.2010.07.004

26. Smart A. A multi-dimensional model of clinical utility. International Journal for Quality in Health Care; 2006; I8(5). https://doi.org//0.1093/intqhc/mzl034.

27. Trochim, W.M. The Research Methods Knowledge Base, 2nd Edition. Retrieved from: http://www.socialresearchmethods.net/kb/> (version current as of 2006). Accessed 15 October 2017.

28. Braun V, Clarke V. Successful qualitative research: A practical guide for beginners. Sage; 2013 . Retrieved from https://www.researchgate.net/publication/256089360_Successful_ Qualitative_Research_A_Practical_Guide_for_Beginners -analysis/. Accessed 16 March $201 \overline{8}$.

29. HPCSA. General Ethical Guidelines for the Health Care Professions. Pretoria: HPCSA; 2016. Available from: https://www.hpcsa. co.za/Uploads/Professional_Practice/Conduct\%20\%26\%20Ethics/ Booklet\%201\%20Guidelines\%20for\%20Good\%20Practice\%20 \%20September\%202016.pdf

30. Brown T, Leo M, Austin DW. Discriminant validity of the Sensory Profile in Australian children with autism spectrum disorder. Physical \& Occupational Therapy in Pediatrics. 2008 Jan I;28(3):253-66. https://doi.org//0.1080/01942630802224983

\section{Corresponding Author:}

* Gina Rencken

Email: rencken@ukzn.ac.za 